\title{
Assessment of ORAl1-mediated basal calcium influx in mammary epithelial cells
}

\author{
Diana GF Ross ${ }^{1}$, Chanel E Smart ${ }^{2}$, Iman Azimi', Sarah J Roberts-Thomson and Gregory R Monteith*
}

\begin{abstract}
Background: The entry of calcium ions into mammary gland epithelial cells is one of the least well-understood processes in the transport of calcium into milk during lactation. The store-operated calcium entry channel ORAl1, has been suggested as a potential mechanism for the entry of $\mathrm{Ca}^{2+}$ into mammary gland epithelial cells from the maternal blood supply during lactation. The down regulation of the canonical ORAI1 activator STIM1 during lactation suggests that other known ORAl activators such as STIM2 and SPCA2 may be important during lactation.

Results: Differentiation of HC11 mammary gland epithelial cells was associated with enhanced basal $\mathrm{Ca}^{2+}$ influx. Silencing of Orail abolished this enhancement of $\mathrm{Ca}^{2+}$ influx. Stim2 had a modest effect on $\mathrm{Ca}^{2+}$ influx in this in vitro model of lactation, whereas Stim 1 and Spca2 silencing had no effect. Despite pronounced increases in Spca2 mRNA during lactation there was no change in the generation of the alternative splice product generated by Mist1, which increases during lactation.
\end{abstract}

Conclusions: These studies support the hypothesis that lactation is associated with a remodelling of $\mathrm{Ca}^{2+}$ influx and this is associated with enhancement of basal $\mathrm{Ca}^{2+}$ influx. This enhanced $\mathrm{Ca}^{2+}$ influx appears to occur through the calcium channel Orai1.

\section{Background}

Lactation is the result of the finely orchestrated differentiation of mammary epithelial cells that gives them the ability to secrete milk. Mammary epithelial cells are unique in their ability to differentiate into lactogenic phenotypes and then dedifferentiate back to a quiescent form, in response to steroid and peptide hormones (reviewed by [1]). Milk provides an energy source, proteins and essential nutrients for the neonate, one of the key components of which is calcium $\left(\mathrm{Ca}^{2+}\right)$. The rapid growth of the neonate, particularly the calcification of bones and teeth, places a high demand for $\mathrm{Ca}^{2+}$ in milk. Depending on the species, the concentration of total $\mathrm{Ca}^{2+}$ in milk ranges from 8 to $60 \mathrm{mM}[2]$, a level well above the maternal blood level of total $\mathrm{Ca}^{2+}$. The secretory pathway and the apical plasma membrane play important roles in the transport of $\mathrm{Ca}^{2+}$ into milk [2-4].

Despite the importance of $\mathrm{Ca}^{2+}$ enrichment of milk, only recently have the $\mathrm{Ca}^{2+}$ transporters responsible for the

\footnotetext{
* Correspondence: gregm@uq.edu.au

'School of Pharmacy, The University of Queensland, Pharmacy Australia Centre of Excellence, 20 Cornwall St, Woolloongabba, QLD, Australia Full list of author information is available at the end of the article
}

accumulation of $\mathrm{Ca}^{2+}$ into milk begun to be identified. The best-characterized protein involved in the enrichment of milk with $\mathrm{Ca}^{2+}$ is the plasma membrane $\mathrm{Ca}^{2+}$ ATPase isoform 2 (PMCA2). This calcium efflux pump has a very restricted tissue expression and is present in specific parts of the brain and the inner ear [5-8]. PMCA2 is markedly up regulated during lactation, particularly splice variant PMCA2bw [5-7,9], which localizes to the apical membrane of secretory cells $[10,11]$. PMCA2 null mice show a $60 \%$ reduction in milk $\mathrm{Ca}^{2+}$ content, providing direct evidence for the role of PMCA2 in the apical transport of $\mathrm{Ca}^{2+}$ into milk during lactation [12].

The sequestration of $\mathrm{Ca}^{2+}$ into the secretory pathway during lactation appears to occur via the Golgi localized pump - secretory pathway $\mathrm{Ca}^{2+}$-ATPase isoform 2 (SPCA2). Like PMCA2, SPCA2 has a restricted tissue distribution [13] and is significantly up regulated during lactation [14]. SPCA2 may also have a dual role in lactation due to its $\mathrm{Mn}^{2+}$ pumping ability [15]. Both $\mathrm{Ca}^{2+}$ and $\mathrm{Mn}^{2+}$ are essential for enzymes necessary for the correct post-translational modification of milk proteins and lactose production [16]. 
Several different $\mathrm{Ca}^{2+}$ permeable ion channels are proposed as the mechanism by which $\mathrm{Ca}^{2+}$ enters the mammary epithelial cell from the maternal blood supply during lactation. Calcium channels suggested as involved in this pathway include TRPV5 and TRPV6 [17,18]. However, recent studies suggest that the Orail calcium channel may be responsible for calcium influx into the mammary epithelial cell during lactation, since Orai1 mRNA levels increase in the mouse mammary gland during lactation [19]. Indeed, Orai1 is at the basolateral membrane in mammary epithelial cells [20]. ORAI1 is the canonical mechanism for store operated calcium entry (SOCE). SOCE is the activation of calcium influx into the cell upon the depletion of intracellular stores of $\mathrm{Ca}^{2+}$. Such a mechanism could be a powerful feedback loop to balance demand (the transport of $\mathrm{Ca}^{2+}$ into milk) with supply (the influx of $\mathrm{Ca}^{2+}$ into the mammary gland epithelial cell). Endoplasmic $\mathrm{Ca}^{2+}$ store level depletion is detected by STIM proteins; upon Endoplasmic Reticulum (ER) $\mathrm{Ca}^{2+}$ depletion, STIM proteins oligomerize and localize to ER-plasma membrane positions where they activate ORAI channels and promote SOCE [21-26]. The Orail isoform of ORAI channels is up regulated in mammary gland tissue samples taken from mice at lactation [19]. However, levels of the canonical Orai1 activator Stim1 decline during lactation. The related isoform Stim2 is suggested as the possible mechanism of activation of Orail during lactation as this isoform does not decrease during lactation and is linked to the regulation of basal $\mathrm{Ca}^{2+}$ influx [21]. Studies identifying the carboxyl terminal of SPCA2 as an activator of ORAI1 and the interaction between SPCA2 and ORAI1 in MCF-7 breast cancer cells, suggests that the up regulation of SPCA2 may not only serve to promote $\mathrm{Ca}^{2+}$ secretion during lactation, but also as an activator of $\mathrm{Ca}^{2+}$ influx. However, this has not been assessed in models of lactation. Another aspect of SPCA2 that has not yet been assessed in lactation is the role of the transcription factor MIST1, which is important in mammary gland development [27]. A novel, truncated form of Spca2 was identified in Mist1-/- mice, but the presence of this form of Spca2 and its potential role in the regulation of $\mathrm{Ca}^{2+}$ transport during lactation has not been assessed. In these studies we used the HC11 model to further explore $\mathrm{Ca}^{2+}$ influx in mammary gland epithelial cells and to define the potential role of MIST1 regulation of Spca2 splicing during mammary gland development.

\section{Results}

Basal $\mathrm{Ca}^{2+}$ influx is increased in differentiated $\mathrm{HC} 11$ cells $\beta$-Casein is a known marker of lactogenesis in mammary epithelial cells necessary for the binding of calcium into micelles [2] and is a reliable marker in HC11 cells to show differentiation. HC11 cells were treated to induce differentiation to a lactogenic phenotype (as described in methods), which was assessed by the expression of $\beta$ casein at the mRNA level. Figure 1A demonstrates the increase in $\beta$-casein mRNA through real-time RT-PCR in differentiated cells in comparison to cells kept in maintenance media in a proliferative non-differentiated state.

Calcium assays were conducted to assess changes in basal $\mathrm{Ca}^{2+}$ influx in response to differentiation of $\mathrm{HC} 11$

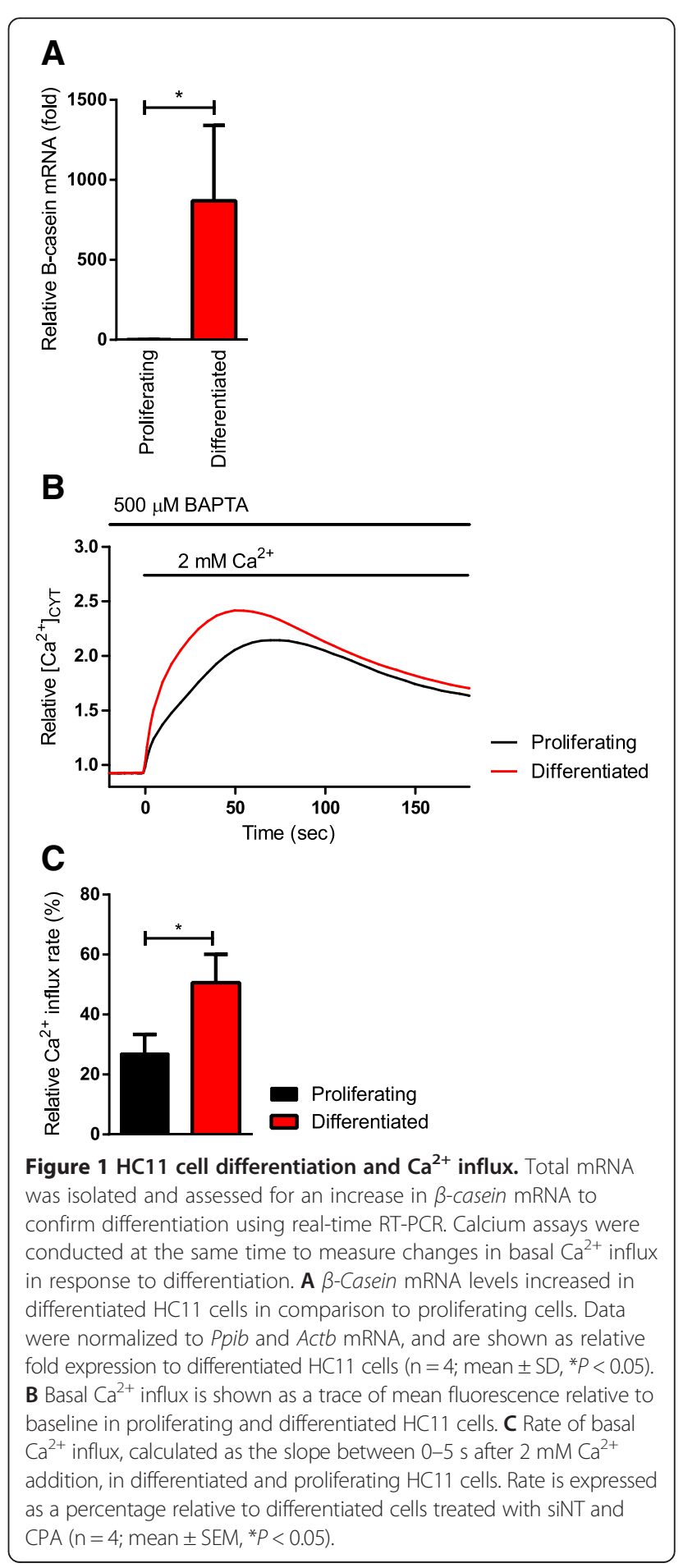


cells. Basal $\mathrm{Ca}^{2+}$ influx (assessed by the rate of $\mathrm{Ca}^{2+}$ increase upon addition of extracellular $\mathrm{Ca}^{2+}$ ) significantly increased in differentiated cells (Figure $1 \mathrm{~B}$ and $\mathrm{C}$ ) indicating that an increase in basal $\mathrm{Ca}^{2+}$ influx accompanies $\beta$-casein induction and may be a characterizing feature of the changes associated with lactation. We then sought to determine the role of Orail in this enhancement of $\mathrm{Ca}^{2+}$ influx.

\section{Enhanced basal $\mathrm{Ca}^{2+}$ influx in differentiated $\mathrm{HC} 11$ cells is abolished by siRNA for Orai1}

The effect of siRNA to Orai1 (siOrai1) on basal $\mathrm{Ca}^{2+}$ influx in proliferative and differentiated $\mathrm{HC} 11$ cells was assessed. Orail silencing eliminated the augmentation of basal $\mathrm{Ca}^{2+}$ influx associated with differentiation (Figure 2A and $\mathrm{B})$ suggesting that Orail mediates this augmentation in differentiated $\mathrm{HC} 11$ cells.

Stim1 silencing did not affect the augmented basal $\mathrm{Ca}^{2+}$ influx in differentiated HC11 cells (Figure 3A and B). Treatment of HC11 cells with siStim 2 did not produce a significant inhibition of $\mathrm{Ca}^{2+}$ influx in differentiated cells (Figure 4A and B), however the significant increase in $\mathrm{Ca}^{2+}$ influx between differentiated and proliferating $\mathrm{HC} 11$ cells was abolished when cells were treated with siStim2 (Figure 4B). These results suggest a major role for Orai1 in $\mathrm{Ca}^{2+}$ influx in the $\mathrm{HC} 11$ model, with a modest role for Stim2.

Spca2 in HC11 cells and mouse mammary tissue samples Truncated and full length Spca2 transcripts [28] were assessed in proliferating and differentiated HC11 cells by comparing mRNA levels measured at exons 15-16 versus exons 26-27, (Figure 5A) and also in mouse mammary tissue samples isolated from virgin, mid pregnancy and lactation stages (Figure 5C). Spca2 mRNA did not increase in the in vitro $\mathrm{HC} 11$ model and there was no change in the proportion of truncated versus full length transcripts (Figure 5A). However, Spca2 mRNA did increase during lactation, although there was no significant difference between the different exon spanning regions of Spca2 indicating that no truncated Spca2 was present in any of the samples. We also assessed the levels of the transcription factor Mist1, which is speculated to regulate Spca2 expression in pancreatic acinar cells [28], and found that Mist1 levels did not significantly change in differentiated $\mathrm{HC} 11$ cells (Figure 5B). However, there was a trend of increased Mist1 levels in mammary gland tissue samples from the lactating mice (Figure 5D) as previously described [27]. Mist1 has been detected in differentiated SCp2 cells [27]. Spca2 silencing had no

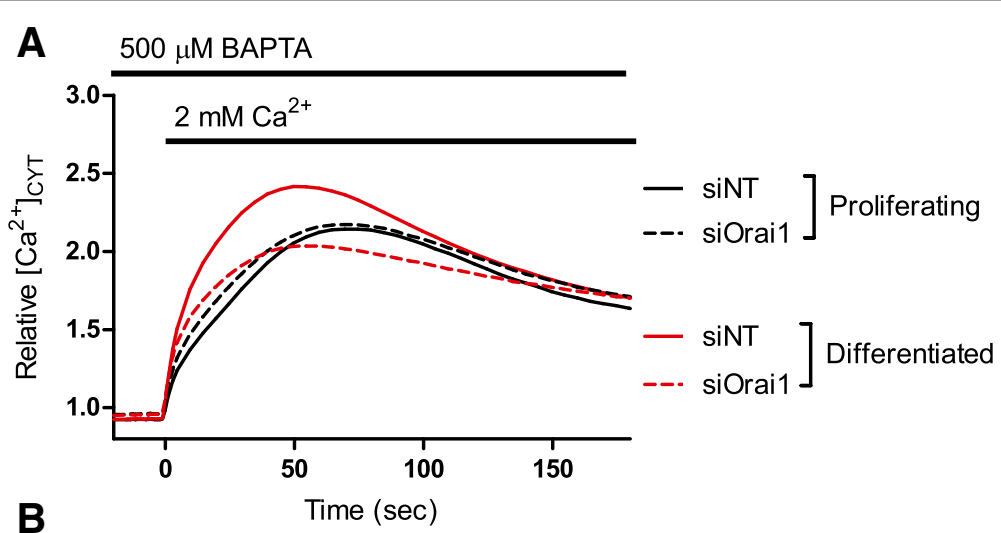

B

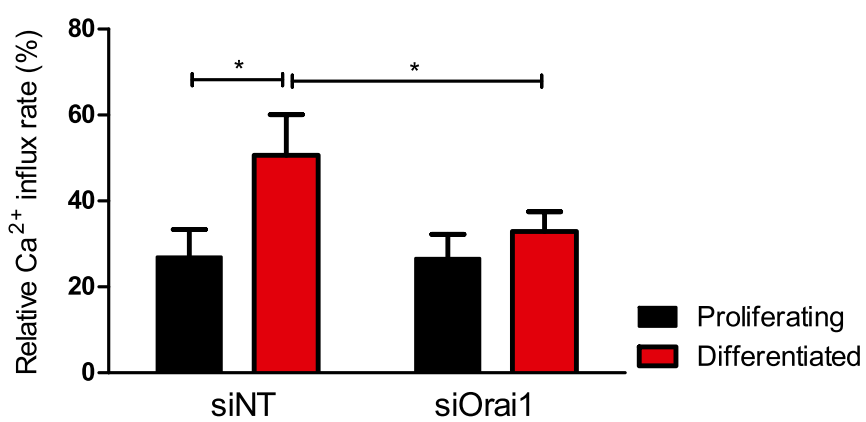

Figure 2 Effect of Orai1 silencing on basal $\mathrm{Ca}^{2+}$ in $\mathrm{HC} 11$ cells. Calcium assays were conducted to assess basal $\mathrm{Ca}^{2+}$ influx in proliferating and differentiated $\mathrm{HC} 11$ cells treated with siNT or siOrai1. A Basal $\mathrm{Ca}^{2+}$ influx is shown as a trace of mean fluorescence relative to baseline in proliferating and differentiated $\mathrm{HC} 11$ cells treated with siNT and siOrai1. B Rate of basal $\mathrm{Ca}^{2+}$ influx, calculated as the slope between $0-5 \mathrm{~s}$ after $2 \mathrm{mM} \mathrm{Ca}^{2+}$ addition, in differentiated and proliferating $\mathrm{HC} 11$ cells with siNT or siOrai1. Rate is expressed as a percentage relative to differentiated cells treated with siNT and CPA $\left(n=4\right.$; mean \pm SEM, $\left.{ }^{*} P<0.05\right)$. 


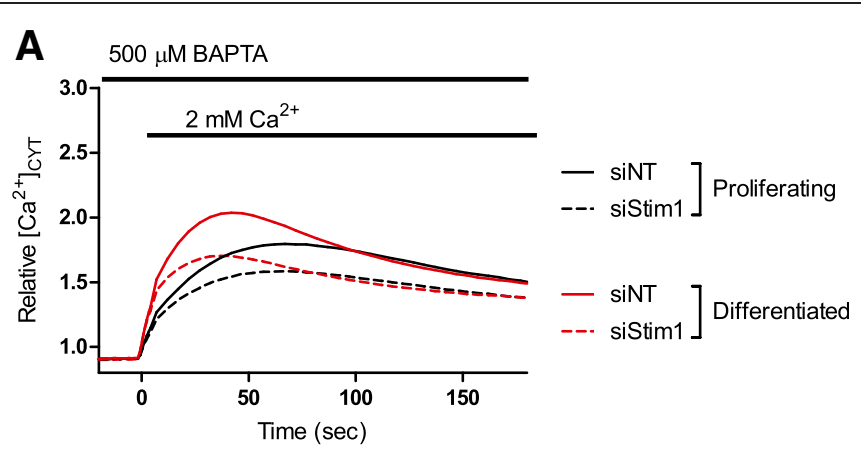

B

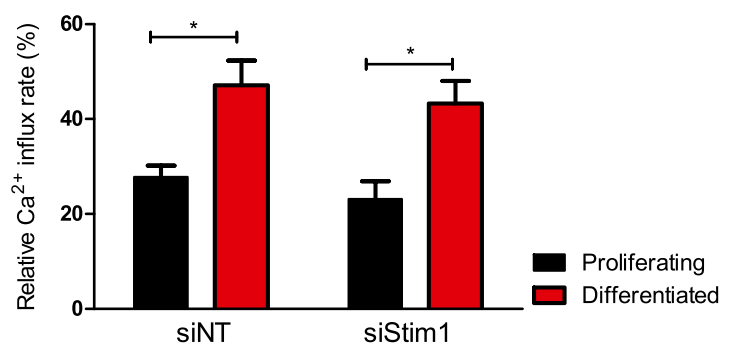

Figure 3 Effect of Stim 1 silencing on basal $\mathrm{Ca}^{2+}$ in $\mathrm{HC} 11$ cells. Calcium assays were conducted to assess the basal $\mathrm{Ca}^{2+}$ influx in proliferating and differentiated $\mathrm{HC} 11$ cells treated with siNT or siStim1. A Basal $\mathrm{Ca}^{2+}$ influx is shown as a trace of mean fluorescence relative to baseline in proliferating and differentiated $\mathrm{HC} 11$ cells treated with siNT and siStim1. B Rate of basal $\mathrm{Ca}^{2+}$ influx, calculated as the slope between 0-5 s after $2 \mathrm{mM} \mathrm{Ca}^{2+}$ addition, in differentiated and proliferating $\mathrm{HC} 11$ cells with siNT or siStim1. Rate is expressed as a percentage relative to differentiated cells treated with siNT and CPA $\left(n=4\right.$; mean \pm SEM, $\left.{ }^{*} P<0.05\right)$.
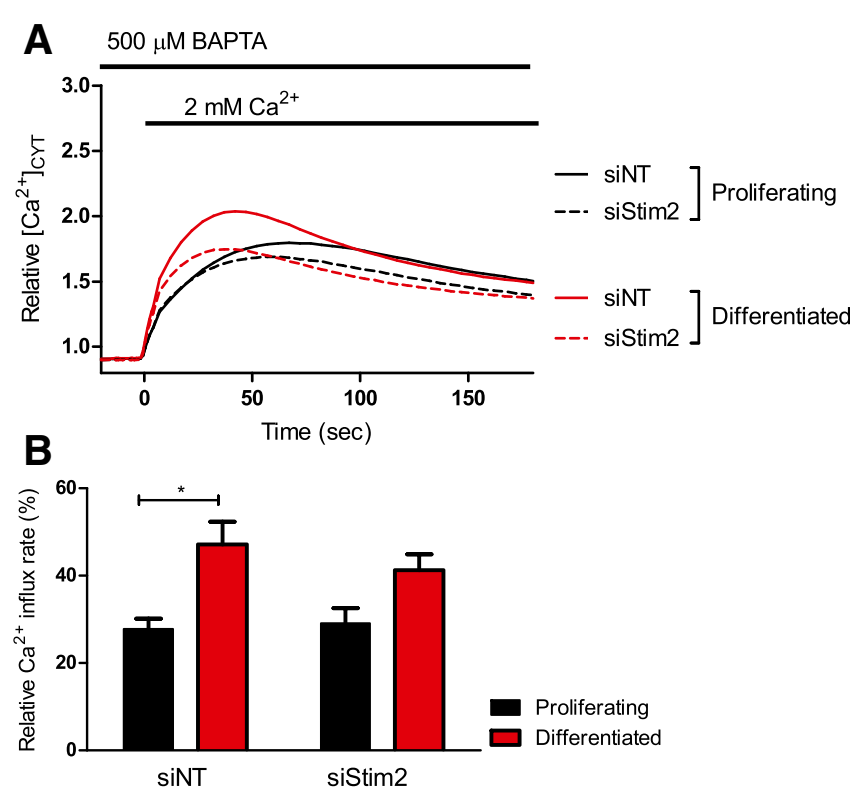

Figure 4 Effect of Stim2 silencing on basal $\mathrm{Ca}^{2+}$ in $\mathrm{HC} 11$ cells. Calcium assays were conducted to assess the basal $\mathrm{Ca}^{2+}$ influx in proliferating and differentiated $\mathrm{HC} 11$ cells treated with siNT or siStim2. A Basal $\mathrm{Ca}^{2+}$ influx is shown as a trace of mean fluorescence relative to baseline in proliferating and differentiated $\mathrm{HC} 11$ cells treated with siNT and siStim2. B Rate of basal $\mathrm{Ca}^{2+}$ influx, calculated as the slope between 0-5 s after $2 \mathrm{mM} \mathrm{Ca}{ }^{2+}$ addition, in differentiated and proliferating $\mathrm{HC} 11$ cells with siNT or siStim2. Rate is expressed as a percentage relative to differentiated cells treated with siNT and CPA $\left(n=4\right.$; mean \pm SEM, $\left.{ }^{*} P<0.05\right)$. 


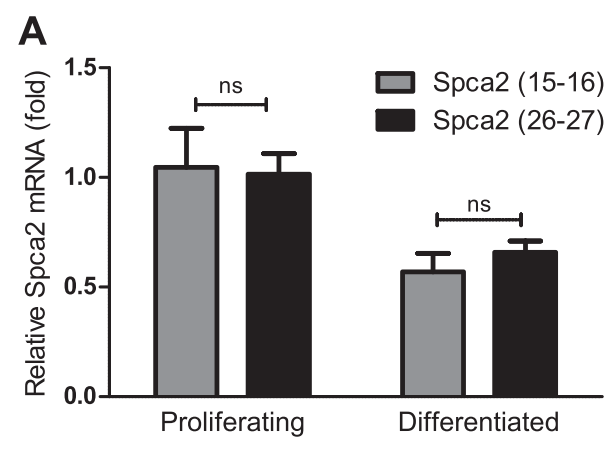

C

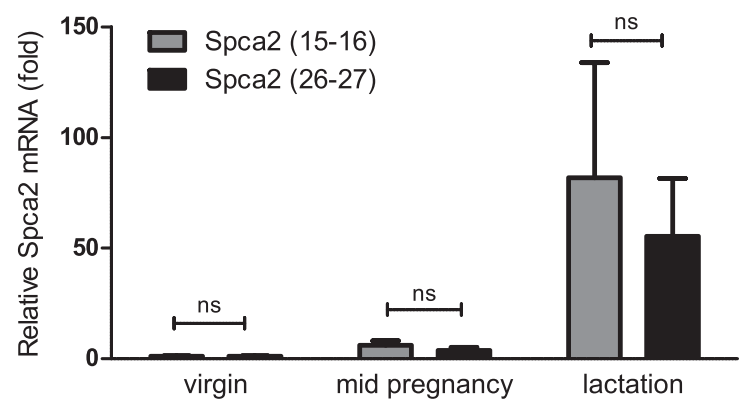

B
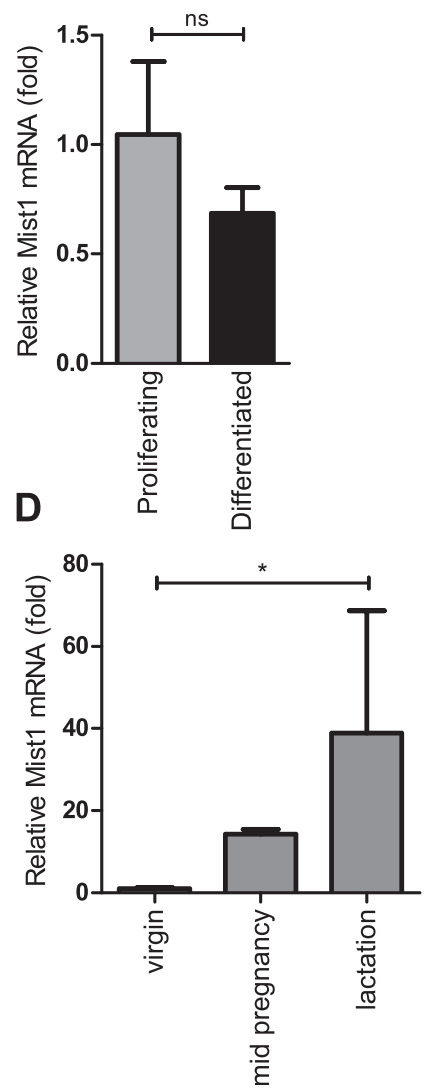

Figure 5 Spca2 mRNA levels and Mist1-mediated truncated Spca2 in lactation. Proliferating and differentiated HC11 cells were assessed for; A. Spca2 mRNA spanning exons 15-16 and 26-27 and B. Mist1 mRNA. Mouse mammary tissues isolated from virgin, mid pregnancy and lactation samples were assessed for; C. Spca2 mRNA spanning exons 15-16 and 26-27 and D. Mist1 mRNA. For all panels $(n=4$; mean $\pm S D$, $n s$ denotes no significant difference $P>0.05)$.

significant effect on basal $\mathrm{Ca}^{2+}$ influx (Figure 6A and B) suggesting that in $\mathrm{HC} 11$ cells Spca2 does not play a role in $\mathrm{Ca}^{2+}$ influx regulation as suggested in other cell types [29].

\section{$\beta$-Casein levels are unchanged with Orai1 silencing}

To determine if the decrease in basal $\mathrm{Ca}^{2+}$ influx was an indirect result of siOrail inhibition of differentiation, $\beta$-casein mRNA levels were measured in siOrai1 treated differentiated HC11 cells. Real-time RT-PCR clearly showed that $\beta$-casein expression was not inhibited in siOrail treated cells (Figure 7). Therefore, although Orail silencing abolishes the induction of increases in $\mathrm{Ca}^{2+}$ influx, it does not inhibit the induction of differentiation.

\section{Orai1 mRNA levels do not change with differentiation}

Given the up regulation of Orail-mediated $\mathrm{Ca}^{2+}$ influx associated with differentiation in $\mathrm{HC} 11$ cells, we assessed changes in the levels of Orail and its known activators. Orail (Figure 8A) mRNA did not significantly change in response to differentiation. However, differentiation was associated with a modest up regulation in Stim1 mRNA (Figure 8B), and a modest down regulation of Stim2 (Figure 8C) and Spca2 (Figure 8D) mRNA levels.

\section{Discussion}

The enrichment of milk with calcium is essential for the growing neonate. Our understanding of the specific calcium channels and pumps that are involved in this process is gradually evolving. However, the area that is least understood in the transport of $\mathrm{Ca}^{2+}$ into milk is the mechanism by which $\mathrm{Ca}^{2+}$ flows from the maternal blood supply into the mammary epithelial cell. In vivo studies implicate store operated $\mathrm{Ca}^{2+}$ entry as the calcium influx pathway involved, with Orail mRNA levels increasing in mouse mammary glands during lactation [19]. Although Orai1 silencing reduces $\mathrm{Ca}^{2+}$ influx in human breast cancer cell lines [19], no studies have directly assessed calcium influx mediated by this pathway in in vitro models of lactation. The possible roles of the Orai1 activators Stim1, Stim2 and Spca2 have also not been assessed through direct assessment of $\mathrm{Ca}^{2+}$ 

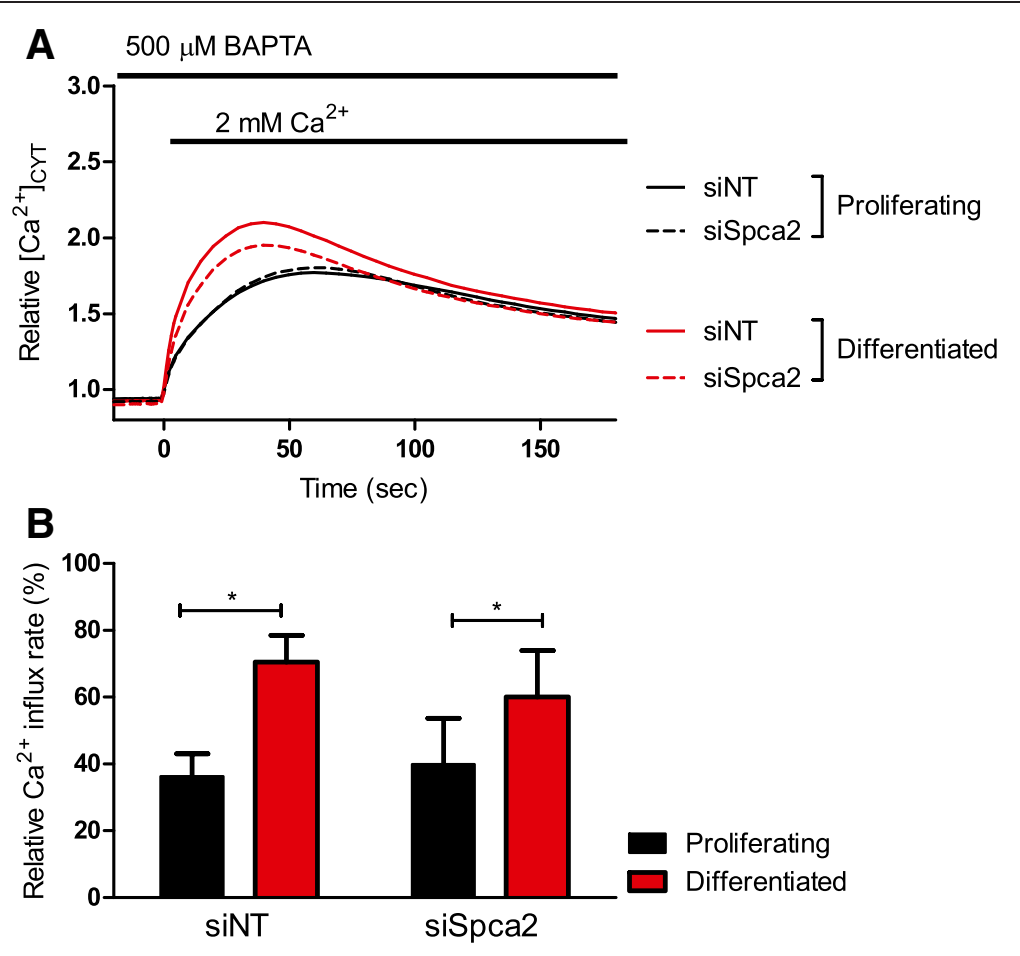

Figure 6 Effect of Spca2 silencing on basal $\mathrm{Ca}^{2+}$ in $\mathrm{HC} 11$ cells. Calcium assays were conducted to assess the basal $\mathrm{Ca}^{2+}$ influx in proliferating and differentiated $\mathrm{HC} 11$ cells treated with siNT or siSpca2. A Basal $\mathrm{Ca}^{2+}$ influx is shown as a trace of mean fluorescence relative to baseline in proliferating and differentiated HC11 cells treated with siNT and siSPCA2. B Rate of basal $\mathrm{Ca}^{2+}$ influx, calculated as the slope between 0-5 s after $2 \mathrm{mM} \mathrm{Ca}^{2+}$ addition, in differentiated and proliferating $\mathrm{HC} 11$ cells with siNT or siSpca2. Rate is expressed as a percentage relative to differentiated cells treated with siNT and CPA $\left(n=4\right.$; mean $\left.\pm S D,{ }^{*} P<0.05\right)$.

influx in mammary gland epithelial cells. These questions were addressed in this study.

HC11 cells are a mouse mammary epithelial cell line derived from COMMA-1D cells, isolated from midpregnant Balb/c mice mammary glands [30,31]. Unlike many other mammary cell models, HC11 cells do not need to be co-cultured with other cell types or with extracellular matrix proteins to help induce lactogenic differentiation [30,32-34], making it suitable for the calcium influx assays used in this study. The expression of $\beta$-casein is essential for $\mathrm{Ca}^{2+}$ accumulation into milk, as a significant amount of calcium is present in casein micelles [2,35]. Moreover, $\beta$-casein is a marker of differentiation in $\mathrm{HC} 11$ cells [36,37].

Differentiation of HC11 cells with lactogenic hormones resulted in a statistically significant increase in basal $\mathrm{Ca}^{2+}$ influx compared to undifferentiated controls. This result is consistent with the hypothesis that lactation is associated with the remodelling of mammary gland epithelial cells to a more $\mathrm{Ca}^{2+}$ permeable phenotype associated with elevated basal $\mathrm{Ca}^{2+}$ influx. This increase in $\mathrm{Ca}^{2+}$ influx is likely to be required to meet the demands of the secretion and efflux of $\mathrm{Ca}^{2+}$ from the mammary gland epithelial cell into milk.

Silencing of Orail demonstrated that this augmented $\mathrm{Ca}^{2+}$ influx is via Orail, since the difference in $\mathrm{Ca}^{2+}$ influx between differentiated and undifferentiated HC11 cells was abolished with the silencing of this calcium channel. Hence, consistent with elevated Orail mRNA levels during lactation, Orai1 appears to be a major contributor of the enhanced basal $\mathrm{Ca}^{2+}$ influx in mammary gland epithelial cells from a lactating host. Orail along with its activator Stim2 are regulators of basal $\mathrm{Ca}^{2+}$ levels [21]. Our studies now suggest that this basal influx pathway is dynamic and may be up regulated during lactation. Future studies should assess how reported regulators of calcium transport during lactation, such as the calcium-sensing receptor, affect Orai1-mediated calcium influx in this and other models [38]).

McAndrews et al. compared 2 day (undifferentiated) and 8 day (with lactogenic hormones - differentiated) HC11 cultures and found elevated Orail mRNA in HC11 cells at 8 days of differentiation [19]. These studies comparing cultures at day 8 with and without lactogenic hormones, suggest that it may be days in culture and/or confluence, which produces an increase in Orail mRNA levels. Lactogenic hormones may be responsible for the enhancement of Orail basal activity in HC11 cells. Orail does not appear to be a key pathway in the differentiation of $\mathrm{HC} 11$ cells given that Orai1 silencing had no effect on $\beta$-casein levels, this is in contrast to 


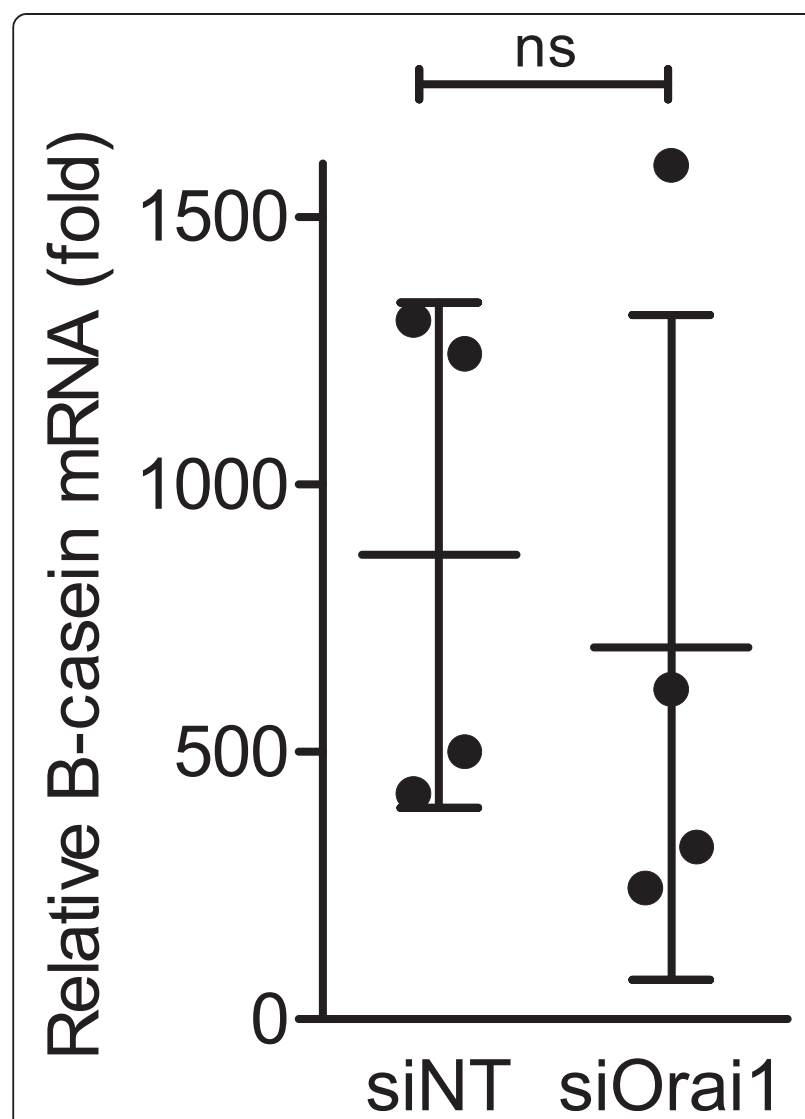

Figure 7 Effect of Orai1 silencing on $\beta$-casein mRNA levels in HC11 cells. $\beta$-Casein mRNA levels were assessed in differentiated HC11 cells treated with NT or Orai1 siRNA. Points on graph represent the average values from three wells across four independent experiments. Data were normalized to Ppib and Actb mRNA, and are shown as relative fold expression to differentiated $\mathrm{HC} 11$ cells. $\left(\mathrm{n}=4\right.$; mean $\pm S D,{ }^{*} P<0.05$; ns denotes no significant difference).

SCp2 cells where mammosphere formation was abolished with shOrai1 treatment [20]. The lack of effects of Orail silencing on $\beta$-casein levels in $\mathrm{HC} 11$ cells indicates that the decrease in basal $\mathrm{Ca}^{2+}$ influx observed when Orail was silenced was not simply a consequence of the inhibition of $\mathrm{HC} 11$ differentiation.

ORAI1 is activated via a number of different mechanisms including the canonical ORAI1 activator and calcium store sensor STIM1, its related isoform STIM2, and SPCA2. STIM2 is proposed as an ORAI1 activator in lactation due to its important role in basal influx in HeLa, HUVEC and HEK293T cells [21]. The maintenance of Stim 2 mRNA levels during lactation [19] and reduction in basal $\left[\mathrm{Ca}^{2+}\right]_{\mathrm{CYT}}$ in differentiated $\mathrm{HC} 11$ cells with Stim2 silencing also support this role. Other mechanisms of ORAI1 activation during lactation have also been proposed, such as calcium store independent activation by SPCA2 [29]. Specific domains of SPCA2 protein activate ORAI1 [29], and Spca2 mRNA levels are pronouncedly increased during lactation [14]. Activation of ORAI1 by SPCA2 may allow the demand for $\mathrm{Ca}^{2+}$ sequestration via this secretory pathway $\mathrm{Ca}^{2+}$ pump to promote the supply of $\mathrm{Ca}^{2+}$ through augmentation of $\mathrm{Ca}^{2+}$ influx. However, in these studies, and in contrast to Orai1 silencing, neither Stim1 nor Spca2 silencing abolished the augmentation of $\mathrm{Ca}^{2+}$ influx induced by differentiation of HC11 mammary epithelial cells. It could be that some in vitro systems have calcium influx pathways that are more sensitive to spca2 dependent modulation of Orai1, such has recently been reported in Scp2 cells [20]. The ability of Stim2 silencing to abolish the enhanced $\mathrm{Ca}^{2+}$ influx associated with differentiation of $\mathrm{HC} 11$ cells, supports previous suggestions that Stim2 is a modulator of Orai1 during lactation, however, the inability of Stim2 silencing to replicate the magnitude of Orai1 silencing suggest other Orail activation mechanisms. Compensatory mechanisms amongst the Orai1 activators Stim1, Stim2 and Spca2 in this model and/or other factors such as the absence of coordinated polarization of mammary gland epithelial cell may be responsible for the results reported here. Further studies using 3D culture models in multiple mammary cell lines models, such as those recently published by Cross et al. in Spc2 cells [20], and in vivo studies with knockout animals are required to ultimately define the specific roles of Stim1, Stim2 and Spca2 and the activation of Orai1 in lactation.

\section{Conclusions}

The influx of $\mathrm{Ca}^{2+}$ across mammary gland epithelial cells is a key step in the supply of $\mathrm{Ca}^{2+}$ to the growing neonate during lactation. These studies using HC11 mammary gland epithelial cells are consistent with recent in vivo studies of mRNA levels during mammary gland development suggesting that Orail is an important pathway of $\mathrm{Ca}^{2+}$ influx during lactation.

\section{Methods}

\section{Cell culture}

Mouse mammary epithelial cells (HC11) were propagated in maintenance media containing Roswell Park Memorial Institute (RPMI)-1640 Medium (R8757, Sigma Aldrich) supplemented with $10 \%$ fetal bovine serum (FBS) and $10 \mathrm{mg} / \mathrm{mL}$ bovine insulin, as previously described [19]. Cells were maintained in a $37^{\circ} \mathrm{C}$, humidified $5 \% \mathrm{CO}_{2}$, 95\% air incubator. Cell lines were routinely tested for mycoplasma contamination.

\section{HC11 cell differentiation and cell culture treatments}

HC11 cells were seeded at 3500 cells/well into 96 well plates in maintenance media containing $10 \mathrm{ng} / \mathrm{mL}$ murine EGF. Fresh maintenance media with EGF was added at $24 \mathrm{~h}$ and siRNA treatment performed at $48 \mathrm{~h}$ in the presence of EGF and insulin. Following $24 \mathrm{~h}$ siRNA treatment 

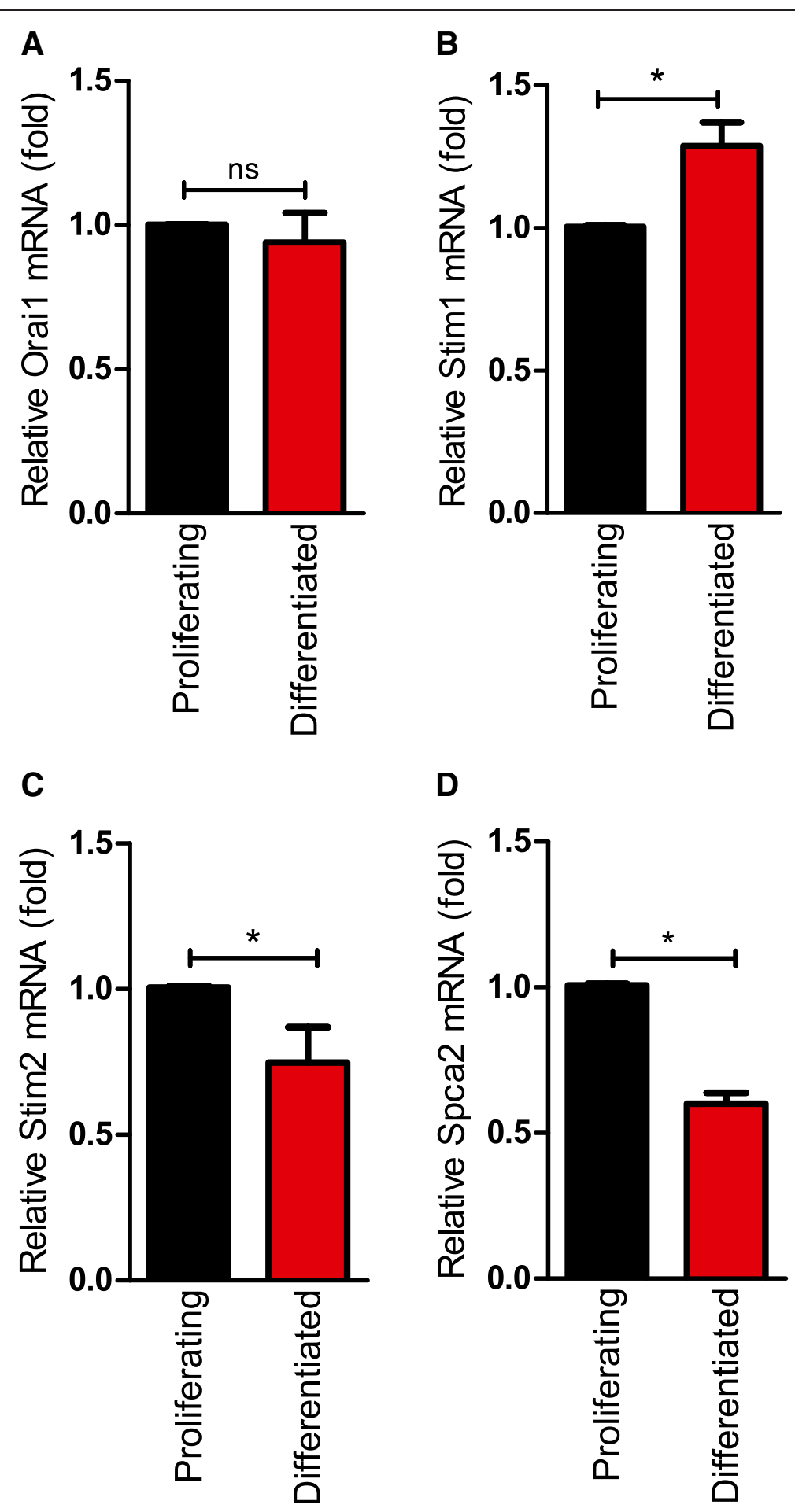

D

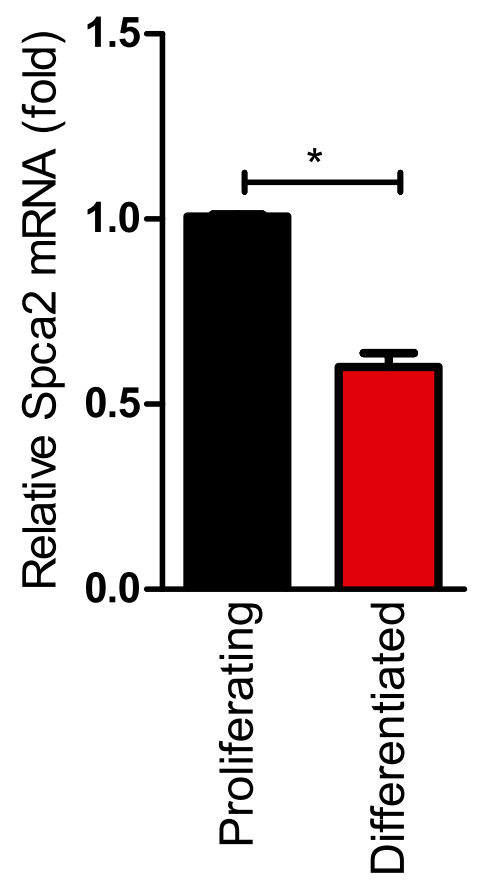

Figure 8 Effect of differentiation in HC11 cells on mRNA expression of Orai1, Stim1, Stim2 and SPCA2. HC11 cells were treated to induce differentiation or kept in maintenance media to keep them proliferating. Total mRNA was isolated $144 \mathrm{~h}$ post plating and assessed for Orail (A), Stim1 (B), Stim2 (C) and Spca2 (D) levels. Data were normalized to Ppib and Actb mRNA, and are shown as relative fold expression to differentiated HC11 cells. ( $n=4$; mean $\pm S D$, ${ }^{*} P<0.05$, ns denotes no significant difference). 
media was replaced with maintenance media for $24 \mathrm{~h}$. After $48 \mathrm{~h}$ siRNA treatment, media was replaced with either maintenance media supplemented with $1 \mu \mathrm{M}$ dexamethasone and $5 \mu \mathrm{g} / \mathrm{mL}$ ovine prolactin (L6520, Sigma Aldrich) to differentiate the cells, or with maintenance media for proliferating non-differentiated cells. Fresh maintenance media was added at $24 \mathrm{~h}$ with or without dexamethasone and prolactin, as appropriate. Calcium assays were performed $144 \mathrm{~h}$ post plating. RNA was isolated from cells after calcium measurements were taken. Experiments were conducted in triplicate wells and all experiments were performed independently on four occasions.

\section{siRNA transient transfection}

siRNA transfection was performed using Dharmacon ONTARGETplus SMARTpool $^{\text {TM }}$ siRNA (100 nM), comprising a pool of four siRNA sequences rationally designed with dual strand modification and use of an algorithm to reduce seed region matches. DharmaFECT1 transfection reagent (catalogue number T-2001-01) was used $(0.4 \mu \mathrm{L} /$ well) as per the manufacturer's instructions. The following Dharmacon On-TARGETplus SMARTpool ${ }^{\mathrm{TM}}$ mouse siRNAs were used in this study: non-targeting (D-001810-1005), Orai1 (L-056431-02-005), Stim1 (L-062376-00-0005), Stim2 (L-055069-01-0005) and Spca2 (L-065820-01-005). siRNA knockdown was checked using real-time RTPCR for each experimental plate $24 \mathrm{~h}$ post treatment (Additional file 1).

\section{Calcium assays}

Calcium assays were performed using a fluorometric imaging plate reader $\left(\right.$ FLIPR $^{\text {TETRA }}$, Molecular Devices Corporation) using the no-wash cytosolic free calcium PBX $\mathrm{Ca}^{2+}$ Assay Kit (BD Biosciences) as previously described [39]. Cells were seeded in 96 well black-walled plates (Corning). Intracellular $\mathrm{Ca}^{2+}$ measurements were performed with an excitation intensity of 470-495 nm and a 515-575 nm emission filter. Fluorescent values were normalized to the starting fluorescence and were expressed as relative $\left[\mathrm{Ca}^{2+}\right]_{\mathrm{CYT}}$. The slope of the curve was calculated based on points at 0 to $5 \mathrm{~s}$ post $\mathrm{Ca}^{2+}$ addition and were expressed as a percentage relative to the average readings for differentiated cells treated with non-targeting siRNA with maximum Orai1-mediated $\mathrm{Ca}^{2+}$ influx induced by $10 \mu \mathrm{M}$ cyclopiazonic acid (CPA).

\section{Real-time RT-PCR}

Total RNA was isolated using the RNeasy Plus mini kit (Qiagen) as per the manufacturer's instructions, and mRNA was quantitated using real time RT-PCR and a 7500 real time PCR system (Applied Biosystems). Mouse mammary tissues were obtained as previously described [19]. Mouse $\beta$-casein (Mm00839664_m1),
Orai1 (Mm00774349_m1), Spca2 (Mm01242899_m1), Spca2 exon 15-16 (Mm01242904_m1), Spca2 exon 26-27 (Mm01242916_m1), Mist1 (Mm00487695_m1), Stim1 (Mm00486423_m1), and Stim2 (Mm01223102_m1) were amplified using the $\operatorname{TaqMan}^{\circ}$ gene expression assays, and the data normalized to Ppib (Mm00478295_m1) and Actb (Mm01205647_g1). Data were analyzed using the comparative $C_{t}$ method as described previously [40]. All data were normalized to proliferating $\mathrm{HC} 11$ cells treated with nontargeting siRNA. Data are shown as mean plus SD $(n=4)$.

\section{Statistical analysis}

All experimental treatments were conducted as three wells per experimental plate in quadruplicate. Results for the slope are presented as standard error of the mean (SEM) and statistical comparisons were performed using a two-way RM ANOVA matching by rows with a Bonferroni multiple comparison post-test. Real-time RTPCR results are presented as standard deviation (SD) and were analyzed for significance using a paired t-test. All statistical analyzes were conducted using Prism Graph Pad, Version 5.04, Berkeley, CA, and significance was demonstrated at $\mathrm{P}<0.05$ where appropriate.

\section{Additional file}

Additional file 1: HC11 cells were treated with siRNA for Orai1 (A.) Stim1 (B.), Stim2 (C.) and Spca2 (D.). Total mRNA was isolated $24 \mathrm{~h}$ after siRNA treatment before real-time. RT-PCR for assessment of gene silencing. Data were normalised to Ppib and ActB mRNA, and are shown as relative fold expression to siNT treated $\mathrm{HC1} 1$ cells ( $n=4$; mean +/- SD, $\left.{ }^{*} P<0.05\right)$.

Competing interests

The authors declare that they have no competing interests.

\section{Authors' contributions}

DR helped design and performed experiments and data analysis and wrote the paper. CS contributed to the writing of the paper and preparation of lactation samples. IA contributed to the writing and revision of the paper. SRT designed experiments and contributed to the data analysis and writing of the paper. GM designed experiments, and contributed to analysis of the data and wrote the paper. All authors read and approved the final manuscript.

\section{Acknowledgements}

We would like to thank Prof. Melissa Brown from the University of Queensland, School of Chemistry and Molecular Biosciences, for providing the mammary tissue samples used in the project. This work was supported by National Health and Medical Research Council Grant 631347.

\section{Author details}

${ }^{1}$ School of Pharmacy, The University of Queensland, Pharmacy Australia Centre of Excellence, 20 Cornwall St, Woolloongabba, QLD, Australia.

${ }^{2}$ University of Queensland Centre for Clinical Research (UQCCR), Building 71/918 Royal Brisbane and Women's Hospital, Herston, QLD 4029, Australia.

Received: 20 November 2013 Accepted: 9 December 2013

Published: 20 December 2013 


\section{References}

1. Borellini F, Oka T: Growth control and differentiation in mammary epithelial cells. Environ Health Perspect 1989, 80:85-99.

2. Neville MC: Calcium secretion into milk. J Mammary Gland Biol 2005, 10:119-128

3. Shennan DB, Peaker M: Transport of milk constituents by the mammary gland. Physiol Rev 2000, 80:925-951.

4. McManaman $\mathrm{JL}$, Neville MC: Mammary physiology and milk secretion. Adv Drug Deliv Rev 2003, 55:629-641.

5. Reinhardt TA, Filoteo AG, Penniston JT, Horst RL: Ca2 + -ATPase protein expression in mammary tissue. Am J Physiol-Cell Ph 2000, 279:C1595-C1602.

6. Reinhardt TA, Horst RL: Ca2 + -ATPases and their expression in the mammary gland of pregnant and lactating rats. Am J Physiol 1999, 276:C796-C802.

7. Strehler EE, Treiman M: Calcium pumps of plasma membrane and cell interior. Curr Mol Med 2004, 4:323-335.

8. Grover AK, Khan I: Calcium pump isoforms: diversity, selectivity and plasticity. Review article. Cell Calcium 1992, 13:9-17.

9. VanHouten JN, Neville MC, Wysolmerski JJ: The calcium-sensing receptor regulates plasma membrane calcium adenosine triphosphatase isoform 2 activity in mammary epithelial cells: a mechanism for calciumregulated calcium transport into milk. Endocrinology 2007, 148:5943-5954.

10. Chicka MC, Strehler EE: Alternative splicing of the first intracellular loop of plasma membrane Ca2 +-ATPase isoform 2 alters its membrane targeting. J Biol Chem 2003, 278:18464-18470.

11. Grati M, Aggarwal N, Strehler EE, Wenthold RJ: Molecular determinants for differential membrane trafficking of PMCA1 and PMCA2 in mammalian hair cells. J Cell Sci 2006, 119:2995-3007.

12. Reinhardt TA, Lippolis JD, Shull GE, Horst RL: Null mutation in the gene encoding plasma membrane Ca2 +-ATPase isoform 2 impairs calcium transport into milk. J Biol Chem 2004, 279:42369-42373.

13. Xiang $M$, Mohamalawari $D$, Rao R: A novel isoform of the secretory pathway $\mathrm{Ca} 2+, \mathrm{Mn}(2+)$-ATPase, hSPCA2, has unusual properties and is expressed in the brain. J Biol Chem 2005, 280:11608-11614.

14. Faddy HM, Smart CE, Xu R, Lee GY, Kenny PA, Feng M, Rao R, Brown MA, Bissell MJ, Roberts-Thomson SJ, Monteith GR: Localization of plasma membrane and secretory calcium pumps in the mammary gland. Biochem Biophys Res Commun 2008, 369:977-981.

15. Vanoevelen J, Dode L, Van Baelen K, Fairclough RJ, Missiaen L, Raeymaekers L, Wuytack F: The secretory pathway Ca2+/Mn2 +-ATPase 2 is a Golgilocalized pump with high affinity for Ca2+ ions. J Biol Chem 2005 280:22800-22808.

16. Lee WJ, Monteith GR, Roberts-Thomson SJ: Calcium transport and signaling in the mammary gland: targets for breast cancer. Biochim Biophys Acta 2006, 1765:235-255

17. VanHouten JN, Wysolmerski JJ: Transcellular calcium transport in mammary epithelial cells. J Mammary Gland Biol 2007, 12:223-235.

18. Hoenderop JGJ, Nilius B, Bindels RJM: Epithelial calcium channels: from identification to function and regulation. Pflug Arch Eur J Phy 2003 446:304-308.

19. McAndrew D, Grice DM, Peters AA, Davis FM, Stewart T, Rice M, Smart CE, Brown MA, Kenny PA, Roberts-Thomson SJ, Monteith GR: ORAl1-mediated calcium influx in lactation and in breast cancer. Mol Cancer Ther 2011, 10:448-460

20. Cross BM, Hack A, Reinhardt TA, Rao R: SPCA2 Regulates Orai1 Trafficking and Store Independent $\mathrm{Ca}(2+)$ Entry in a Model of Lactation. PLoS One 2013, 8:e67348.

21. Brandman O, Liou J, Park WS, Meyer T: STIM2 is a feedback regulator that stabilizes basal cytosolic and endoplasmic reticulum $\mathrm{Ca} 2+$ levels. Cell 2007, 131:1327-1339.

22. Liou J, Fivaz M, Inoue T, Meyer T: Live-cell imaging reveals sequential oligomerization and local plasma membrane targeting of stromal interaction molecule 1 after Ca2+ store depletion. Proc Natl Acad Sci USA 2007, 104:9301-9306.

23. Liou J, Kim ML, Heo WD, Jones JT, Myers JW, Ferrell JE Jr, Meyer T: STIM is a $\mathrm{Ca} 2+$ sensor essential for $\mathrm{Ca} 2+-$ store-depletion-triggered $\mathrm{Ca} 2+$ influx. Curr Biol 2005, 15:1235-1241.

24. Wu MM, Buchanan J, Luik RM, Lewis RS: Ca2+ store depletion causes STIM1 to accumulate in ER regions closely associated with the plasma membrane. J Cell Biol 2006, 174:803-813.
25. Xu P, Lu J, Li Z, Yu X, Chen L, Xu T: Aggregation of STIM1 underneath the plasma membrane induces clustering of Orai1. Biochem Biophys Res Commun 2006, 350:969-976.

26. Luik RM, Wu MM, Buchanan J, Lewis RS: The elementary unit of storeoperated Ca2+ entry: local activation of CRAC channels by STIM1 at ERplasma membrane junctions. J Cell Biol 2006, 174:815-825.

27. Zhao Y, Johansson C, Tran T, Bettencourt R, Itahana Y, Desprez P-Y, Konieczny SF: Identification of a basic helix-loop-helix transcription factor expressed in mammary gland alveolar cells and required for maintenance of the differentiated state. Mol Endocrinol 2006, 20:2187-2198.

28. Garside VC, Kowalik AS, Johnson CL, DiRenzo D, Konieczny SF, Pin CL: MIST1 regulates the pancreatic acinar cell expression of $A t p 2 c 2$, the gene encoding secretory pathway calcium ATPase 2. Exp Cell Res 2010, 316:2859-2870.

29. Feng M, Grice DM, Faddy HM, Nguyen N, Leitch S, Wang Y, Muend S, Kenny PA, Sukumar S, Roberts-Thomson SJ, et al: Store-independent activation of Orai1 by SPCA2 in mammary tumors. Cell 2010, 143:84-98.

30. Ball RK, Friis RR, Schoenenberger CA, Doppler W, Groner B: Prolactin regulation of beta-casein gene expression and of a cytosolic 120-kd protein in a cloned mouse mammary epithelial cell line. EMBO J 1988, 7:2089-2095.

31. Danielson KG, Oborn CJ, Durban EM, Butel JS, Medina D: Epithelial mouse mammary cell line exhibiting normal morphogenesis in vivo and functional differentiation in vitro. Proc Natl Acad Sci USA 1984, 81:3756-3760.

32. Streuli CH, Bailey N, Bissell MJ: Control of mammary epithelial differentiation: basement membrane induces tissue-specific gene expression in the absence of cell-cell interaction and morphological polarity. J Cell Biol 1991, 115:1383-1395.

33. Lin CQ, Bissell MJ: Multi-faceted regulation of cell differentiation by extracellular matrix. FASEB J 1993, 7:737-743.

34. Chammas R, Taverna D, Cella N, Santos C, Hynes NE: Laminin and tenascin assembly and expression regulate $\mathrm{HC} 11$ mouse mammary cell differentiation. J Cell Sci 1994, 107(Pt 4):1031-1040.

35. Neville MC, Watters CD: Secretion of calcium into milk: review. J Dairy SCi 1983, 66:371-380

36. Taverna D, Groner B, Hynes NE: Epidermal growth factor receptor, platelet-derived growth factor receptor, and c-erbB-2 receptor activation all promote growth but have distinctive effects upon mouse mammary epithelial cell differentiation. Cell Growth Differ 1991, 2:145-154.

37. Venesio T, Taverna D, Hynes NE, Deed R, MacAllan D, Ciardiello F, Valverius EM, Salomon DS, Callahan R, Merlo G: The int-2 gene product acts as a growth factor and substitutes for basic fibroblast growth factor in promoting the differentiation of a normal mouse mammary epithelial cell line. Cell Growth Differ 1992, 3:63-71.

38. Mamillapalli R, VanHouten J, Dann P, Bikle D, Chang W, Brown E, Wysolmerski J: Mammary-specific ablation of the calcium-sensing receptor during lactation alters maternal calcium metabolism, milk calcium transport, and neonatal calcium accrual. Endocrinology 2013, 154:3031-3042.

39. Grice DM, Vetter I, Faddy HM, Kenny PA, Roberts-Thomson SJ, Monteith GR: Golgi calcium pump secretory pathway calcium ATPase 1 (SPCA1) is a key regulator of insulin-like growth factor receptor (IGF1R) processing in the basal-like breast cancer cell line MDA-MB-231. J Biol Chem 2010, 285:37458-37466.

40. Aung CS, Ye W, Plowman G, Peters AA, Monteith GR, Roberts-Thomson SJ: Plasma membrane calcium ATPase 4 and the remodeling of calcium homeostasis in human colon cancer cells. Carcinogenesis 2009, 30:1962-1969.

doi:10.1186/1471-2121-14-57

Cite this article as: Ross et al: Assessment of ORAl1-mediated basal calcium influx in mammary epithelial cells. BMC Cell Biology 2013 14:57. 